\title{
Research on Calibration Method of the Beam Direction the Point Laser Sensor Based on Standard Ball
}

\author{
Chengwei Li, Jianguo Fang, Jingliang Liu, Fei Liu, Di Li \\ Aviation Key Laboratory of Science and Technology on Precision Manufacturing NanYuan East \\ Road NO.5,FengTai District, BeiJing,China
}

Keywords: laser sensor; beam direction; calibration method;

\begin{abstract}
Non-contact measurement has the characteristics of high speed and high efficiency, which fit the requirements of the measurement of engine blade. According to high-speed and efficient measurement in the condition of high precision, we design a point laser beam direction calibration method based on a standard ball by analysing the mathematical model. By experiment, it proves that the stability and accuracy can meet the requirements
\end{abstract} of blade measurement.

\section{Introduction}

At present, the aero engine blades are measured mainly by the three coordinate measuring machine detection. The low efficiency detection cannot meet the requirements measurement. Each aircraft engine manufacturing plant blade is eager tomeasuring equipment with the high efficiency and the high precision. Professor Zhang Guoxiong of Tianjin University proposed that [1]the technique of non-contact probe, especially optical probe with high efficiency and natural anti-collision design, is one of the most important development direction of the coordinate measuring technology.

To fit the requirement of the high efficiency and high precision measurement of the complex freeform surface, WENZEL company, according to the non-contact probe's characteristics of high speed and high efficiency, launched his non-contact measurement production to meet the precision measurement needs of the measuring machine market in 2010, while the domestic research is still in the verification experiment stage, whose accuracy cannot meet the requirements of precision measurement. Therefore, the launch of the domestic non-contact measuring machine with the precision requirement can meet the demand of the market.

As with the contact type coordinate measuring system, optical coordinate measuring system pays attention to the relative position of the measured object. Contact type measuring system get the message from the position of the guide rail and the direction and radius of the probe. So the difference optical coordinate measuring system (for example, the point laser sensor) also needs to calibrate the direction of the beam and the distance to the objects. Therefore, the optical coordinate measuring system needs linear and optical axis of optical probe calibration. At present, the laser sensor manufacturers already make the linear calibration measured as a key point, before they leave the factory. Therefore, we are concerned more about the calibration of the optical beam direction of the optical sensor.

Engineer David Bunimovich of Israel Nextec company and Professor Zexiao Xie of Ocean University of China respectively [2,3] proposed a non-contact scanning probe calibration method based on multi point iteration. Due to the large number of point cloud data in the calibration process, it is not convenient for practical use of point laser sensor.

Huizheng Zhou, Associate Professor of Science and Technology University, Keqing Lu, student of Zhejiang University, and Yong Liu, senior engineer of AVIC Beijing Precision Engineering Institute for Aircraft Industry respectively $[4,5,6]$ proposed calibration method based on the two intersecting plane of the laser beam. But because the optical probe has a certain angle limitation in the use of the process, a set of calibration planes can't meet the requirement of space optical axis rotation vector calibration, and replacing the calibration block is not conducive to the use of software to automatical and fast calibration. Therefore, we need a calibration method, which can 
be applied to the space of all optical beam. Based on the ball, Jianguo Fang, fellow of AVIC Beijing Precision Engineering Institute for Aircraft Industry proposed[7] calibration method of point laser beam direction based on standard ball. We will research and prove the applicability and accuracy of calibration.

\section{Design and analysis of mathematics model}

In industrial applications, triangulation laser probe is installed on the guide rail, in order to scanning measure. It is usually installed on the $\mathrm{Z}$ axis of the three coordinate measuring machine or CNC machine tools, realizing scanning trajectory through the $\mathrm{X}, \mathrm{Y}$ axis motion, in order to make the measuring sensor reach each measuring position; $\mathrm{Z}$ axis motion makes the measuring head to adapt to the change of the height of the object surface. At the time of measurement, we should not only maintain the measured surface located in the probe measurement range, but also ensure the measured angles $(\theta)$ between the measurement beam direction and the surface normal direction in the allowable range of laser sensor. Therefore, when the measured surface curvature changes, in order to ensure the measuring precision, we need through the rotary table to change the beam directions, to ensure that $\theta$ within the allowable range. Figure 1 is the point laser sensor coordinate measuring machine working principle diagram.

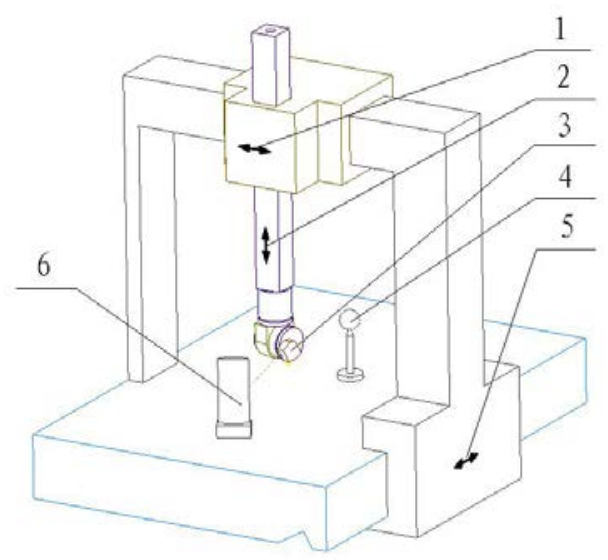

(1-Y axis, 2-Z axis, 3- laser sensor, 4- standard ball, 5-X axis, 6- blade)

Fig. 1 Schematic diagram [7]

We set the optical axis to point laser sensor as a one-dimensional space, and set a optical measuring head read numerical 'spot position 0 ' as the origin of optical axis. In the installation process, the optical axis origin, measuring head rotation center and probe in the machine tool coordinate position (referred to as the probe machine coordinate) are misalignment. This error can be used to represent a vector. We set the error vector $\vec{a}$ as the origin of the optical axis to the rotation center, error vector $\vec{b}$ as the rotation center to the position of machine coordinate, and error vector $\vec{c}$ as the origin of the optical axis to the position of probe machine coordinate. We can get that $\vec{a}+\vec{b}=\vec{c}$. The theoretical model as shown in Figure 2 is obtained. 


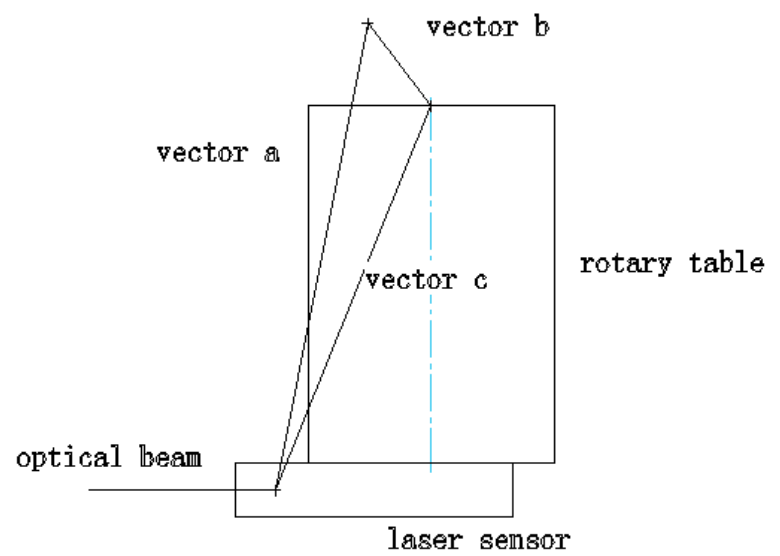

Fig. 2 theoretical models of head installation

Among them, the vector $\vec{b}$ depends only on the position of the rotating shaft and will not change during the process of measurement. So it will not influence the measurement results. Vector $\vec{a}$ will produce equal angle changes when the optical axis direction rotates with the rotary shaft. As the origin of optical axis is not in reality, we cannot get the rotation position changes easily. Therefore, we assume that in the process of calibration and measurement, optical sensor does not rotate. Vector $\vec{a}$ in the process of calibration and measurement the remain unchanged so that it can be ignored in the process of calculation.

Under this premise, the coordinate of the laser measuring spot in the machine toolcoordinate system can be expressed as $(x+1 \cos \alpha, y+l \cos \beta, z+l \cos \gamma)$, among them, $x, y$, and $z$ mean the coordinates of laser sensor in the position of the machine coordinate system. And l means the reading value of the point laser sensor. And $\alpha, \beta$, and $\gamma$ respectively mean the angle between the beam direction in machine coordinate system and three coordinate axis vector. When using the ball as a calibration object, in condition of unknown ball in the machine coordinate system of the position of the $\mathrm{M} 0(\mathrm{x} 0, \mathrm{y} 0, \mathrm{z} 0)$ and the radius of the case of $\mathrm{R}$, we have a total of 7 unknowns. The theoretical model are shown in figure 3.

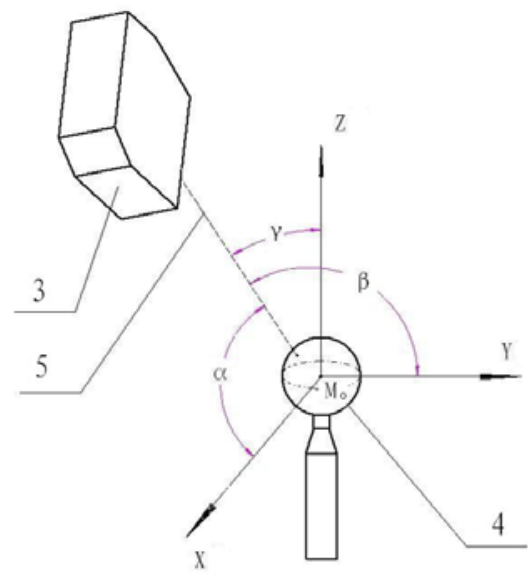

(3- laser sensor, 4- standard ball, 5- beam line)

Figure 3 point laser sensor beam calibration principle [7]

7 groups of measuring data, establish the following equations and solving. $\left[x_{0}-\left(x_{i}+l_{i} \cos \alpha\right)\right]^{2}+\left[y_{0}-\left(y_{i}+l_{i} \cos \beta\right)\right]^{2}+\left[z_{0}-\left(z_{i}+l_{i} \cos \lambda\right)\right]^{2}=r^{2}(1)$

In the formula, (xi, yi, zi) respectively denote the coordinate datas of the laser sensor in the machine coordinate. With a simple derivation, we can know that $(\mathrm{x} 0$-licos $\alpha, \mathrm{y} 0$-licos $\beta, \mathrm{z} 0$ licos $\gamma$ ) is a calibration ball center obtained by reverse translation along the optical beam direction of li, the above equation can also be regarded as to the point of center ( $\left.x_{i}^{\prime}, y_{i}^{\prime}, z_{i}^{\prime}\right)$, on the ball of 
the equations for the ball measuring head coordinates of points on the. When the laser sensor read two point numerical equal, probe position corresponding to the two point of the ball with the same radius in the standard ball.

In order to obtain the standard ball along the optical axis direction reverse migration of new sphere center position (X, Y, Z), 4 equations are needed, with $l_{1}=l_{2}=l_{3}=l_{4}$. We can get the new center $(X, Y, Z)$ by solving the formula when i equals with $1,2,3$, and 4 . We can also get the radius of the ball.

Similarly, with $l_{5}=l_{6}=l_{7}, l_{5}-l_{1}=L$, together with $\cos ^{2} \alpha+\cos ^{2} \beta+\cos ^{2} \lambda=1$, we can infer from formula the direction of the laser beam $(\alpha, \beta, \gamma)$ when i equals with 5,6 , and 7 .

Thus we can get the direction of the laser beam, completing the calibration of the optical axis vector.

\section{Experiment}

3.1 experimental conditions

In this paper, we use Pearl 1298 type measuring machine as the body of the optical measuring machine, whose compensation accuracy is 2.8+L /350 um. Using CD5-85 laser sensor, which has the measuring distance of $85 \mathrm{~mm}$ and the measurement range of $\pm 20 \mathrm{~mm}$, the resolution of $1 \mathrm{um}$, the linearity of $\pm 0.05 \%$. The laser sensor is connected to the NSK motor through the adapter plate. The motor has the absolute position accuracy of 18 " and 1.3" repeat accuracy. We use the stone ball as a calibration object.

3.2 experimental procedures

First of all, through the manual calibration, determining the approximate beam direction and the calibration ball center: 1 Determine the values of the first four points and the post three points; 2 Get the approximate machine coordinate of the seven spot and make sure the first four spot from different circles and the post three spot from different lines; 3 By substitution of the data into the solution formula, get the manual calibration of the optical axis direction and the spherical center; 4 Input the manual calibration data; 5 automatic calibration experiment.

In order to verify the calibration precision, we repeat the calibration experiment and the calibration data are based on the center coordinates and the direction of optical axis we got in the last time. With $l_{1}=l_{2}=l_{3}=l_{4}=70 \mathrm{~mm}$ and $l_{5}=l_{6}=l_{7}=100 \mathrm{~mm}$, we make the beam of the first spot set through the ball center and the remaining six spots on a circular whose central angle is 60 deg. We totally make 31 experiments, and record the experimental results.

\section{Result Analysis}

When the sensor reads the value $l$ and the calibration error is $\theta$, measurement error of single point caused by calibration errors is $2 \times l \sin \frac{\theta}{2}$. If $\theta$ is very small, we can view $\sin \theta$ as $\theta$. Therefore, the measurement error caused by the calibration can be simplified to $l \theta$. In measuring the relative position relationship between two points calibration, if the beam direction does not change, the calibration error $\theta$ is a fixed value. So the error of the relative position depends on the range of the sensor output value.

When we plan the measuring path, though we are not able to keep the output of the laser sensor invariant, namely laser probe measurement path is formed by the measured curve shifting, we can still use $\Delta l$ in the process of measuring path planning to control the error caused by the calibration of non-contact sensor beam direction.

Analyzing the experimental results, each calibration results and the calibration space angle difference between the mean are shown as follows in Figure 4: 


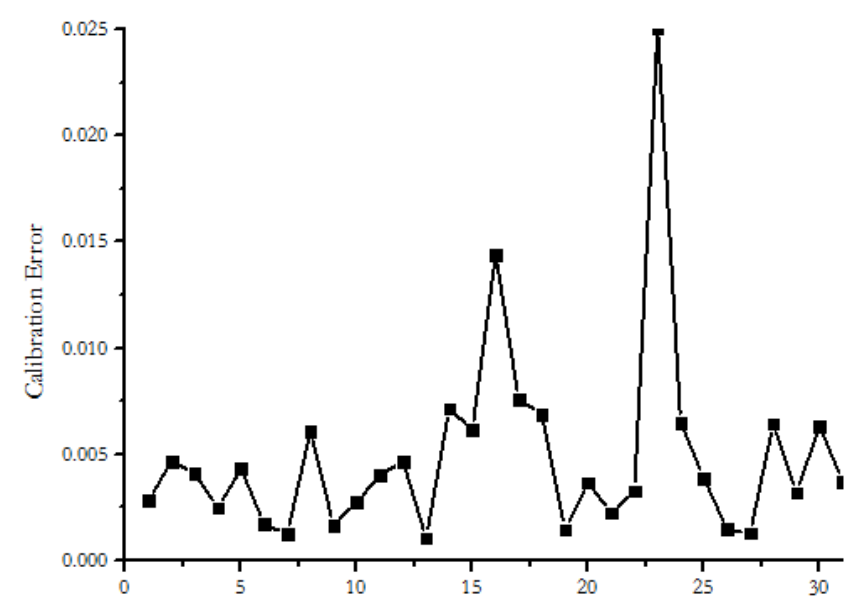

Figure 4 calibration error

In order to ensure the accuracy of the calibration results, we think that the result is subject to the normal distribution variance whose expectation is the truth value and variance is $\sigma^{2}$. According to the chi-square test of the mathematical statistics, we can get a conclusion that the calibration error is less than $0.02 \mathrm{deg}$.

In the course of using non-contact sensor, calibration error will directly effort the measurement results. So the optimization calibration algorithm will directly enhance the measurement precision. According to the error distribution before, seven point standard ball calibration method can provide enough accuracy. Experimental results show that the calibration precision can reach 0.02 degrees, that is to say, the measurement error caused by the calibration can reach $5 \mu \mathrm{m}$ with $15 \mathrm{~mm}$ range of the laser sensor output, which can meet the demand for the calibration of noncontact measurement system of aero engine blades.

\section{Conclusion}

In this paper, we use the laser sensor, the precision rotary table and the three coordinate measuring machine to build a non-contact measurement system. In order to realize the function that the beam direction of laser sensor can be calibration in any direction, we proposed a calibration method based on the standard ball. In the process of calibration, we record the coordinates of the laser sensor, so as to establish the equations with center coordinates and radius, the direction of the beam direction. By solving the equations, we can complete the calibration of the beam direction. Experimental results show that the method proposed in this paper to make the laser beam direction calibration error less than 0.02 degree, which can ensure a high accuracy to meet the requirements of non-contact measurement of aero engine blades.

\section{Acknowledgements}

The authors are grateful for the financial support provided by the National Science and Technology Program of China (2013ZX04011031).

\section{References}

[1]Guoxiong Zhang , The Coordinate Measuring Technology Development Direction of [J] Infrared and Laser Engineering, 2008 April, Vol. thirty-seventh, suppl, in Chinese.

[2]David Bunimovich, Calibration Process for Shape Measurement: U.S,6,199,024 B1[P].2001-0306

[3] Zexiao Xie, The Calibration method of no-contact probe based on Vision. China, 200510044577.3[P].2006-03-01, in Chinese 
[4] Huicheng Zhou, Lizhan Zeng, Jihong Chen. The Direction Calibration of the pointolite's beam. [J]. Chinese Journal of Scientific Instrument, 2004， 25(3)388-391, in Chinese

[5] Keqing Lu, Wen Wang, Zichen Chen. Calibration of Laser Sensor Beam Direction [J]. Optics and Precision Engineering, 2010,14 (4)880-886, in Chinese

[6]Yong Liu, Chao Bi, Ying Gao, et al. Research and application of plane calibration method of optical sensor beam direction. [J]. Measurement and Control, in Chinese Technology 2013, 32 (Suppl):.513-515.

[7] Jianguo Fang, Tan zhang, Liang Fang. A Calibration Method of the Spot-Structured optical probe's spatial position and vector. China, 201310493454.2. in Chinese 